\title{
Style Sheet
}

Expanded Polytetrafluoroethylene Blood Vessel Grafts with Embedded Reactive Oxygen Species (ROS) Responsive Antithrombogenic Drug for Elimination of Thrombosis

Dongfang Wang, Yiyang Xu, Lixia Wang, Xiaofeng Wang, Cuihong Ren, Bo Zhang, Qian Li*, James A. Thomson, and Lih-Sheng Turng*

Keywords: ePTFE, blood vessel grafts, ROS response, smart release, thrombus, bulk coating

\author{
Abbreviations: \\ Abstract: \\ SDBV small-diameter blood vessel \\ ePTFE expanded polytetrafluoroethylene \\ ROS reactive oxygen species \\ ESA ethyl salicylate \\ PCL polycaprolactone \\ NMR nuclear magnetic resonance (spectroscopy) \\ FTIR Fourier-transform infrared (spectroscopy) \\ SEM scanning electron microscopy \\ AFM atomic force microscopy \\ LDH lactate dehydrogenase \\ Introduction and further: \\ ePTFE expanded polytetrafluoroethylene \\ SDBV small-diameter blood vessel \\ ROS reactive oxygen species \\ ESA ethyl salicylate \\ mPEG methoxy poly(ethylene glycol) \\ PCL polycaprolactone \\ NMR nuclear magnetic resonance (spectroscopy) \\ FTIR Fourier-transform infrared (spectroscopy) \\ SEM scanning electron microscopy \\ CF chloroform \\ AFM atomic force microscopy \\ DSC differential scanning calorimetry \\ PBS phosphate-buffered saline \\ PPP platelet-poor plasma \\ PRP platelet-rich plasma \\ LDH lactate dehydrogenase \\ HUVECs human umbilical vein endothelial cells \\ DCM dichloromethane \\ DAPI 4',6-diamidino-2-phenylindole \\ ANOVA analysis of variance
}




\section{Numbers:}

No comma in thousands place $3300 \mathrm{~cm}^{-1}$

$37^{\circ} \mathrm{C}$

$10 \%$

Names and terms:

Abcam, Cambridge, UK

Alfa Aser, USA

Attenuated Total Reflection mode

Bio-Vision

Bruker, Tensor27, Germany

$\mathrm{C}=\mathrm{C}$ bonds

$\mathrm{C}=\mathrm{O}$ bond or bonds

$\mathrm{CaCl}_{2}$

calcein AM

CFTM 568, Biotium, USA

D9542, Sigma-Aldrich

Dataphysics, OCA15, US

Discovery DSC2500, TA, USA

Eclipse Ti, Nikon, Japan

EGMTM-2 BulletKitTM Medium, CC-3162, Thermo Fisher

ethidium homodimer-1

ePTFE-drug

ePTFE-PCL (control)

ESA-oxalyl chloride

ESA-oxalyl chloride-mPEG

ethyl 2-(2-chloro-2-oxoacetoxy) benzoate

glutaraldehyde

${ }^{1} \mathrm{H}$ NMR

hexane

hydrophilicity

Instron 5967, $30 \mathrm{kN}$, USA

JNM EX-400, JEOL, Japan

oxalyl chloride

peroxalate ester

Phalloidin

prodrug

pyruvate

S-3100, SINCO, Korea

SCD40L

Synergy-HT, BioTek

triethylamine hydrochloride

Triton-X

Zeiss, Germany 